Please do not remove this page

RMIT

UNIVERSITY

\title{
Two definitions of the inner product of modes and their use in calculating non-diffuse reverberant sound fields
}

Nolan, Melanie; Davy, John

https://researchrepository.rmit.edu.au/esploro/outputs/9921860818401341/filesAndLinks?institution=61RMIT_INST\&index=null

Nolan, M., \& Davy, J. (2019). Two definitions of the inner product of modes and their use in calculating non-diffuse reverberant sound fields. Journal of the Acoustical Society of America, 145(6), 3330-3340. https://doi.org/10.1121/1.5109662

Document Version: Accepted Manuscript

Published Version: https://doi.org/10.1121/1.5109662

Repository homepage: https://researchrepository.rmit.edu.au

(c) 2019 Acoustical Society of America

Downloaded On 2023/04/26 21:05:58 +1000

Please do not remove this page 
Thank you for downloading this document from the RMIT Research Repository.

The RMIT Research Repository is an open access database showcasing the research outputs of RMIT University researchers.

RMIT Research Repository: http://researchbank.rmit.edu.au/

\section{Citation:}

Nolan, M and Davy, J 2019, 'Two definitions of the inner product of modes and their use in calculating non-diffuse reverberant sound fields', Journal of the Acoustical Society of America, vol. 145, no. 6, pp. 3330-3340.

See this record in the RMIT Research Repository at:

https://researchbank.rmit.edu.au/view/rmit:53966

Version: Accepted Manuscript

Copyright Statement:

(C) 2019 Acoustical Society of America

\section{Link to Published Version:}

https://dx.doi.org/10.1121/1.5109662 
Two definitions of the inner product of modes and their use in calculating non-diffuse reverberant sound fields

$$
\text { Mélanie Nolan a), b) and John L. Davy c), d) }
$$

b) Acoustic Technology, Department of Electrical Engineering,

Technical University of Denmark, Building 352, Ørsteds Plads, DK-2800 Kongens Lyngby, Denmark

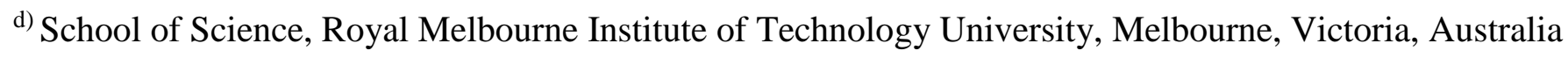

May 6, 2019

Running title: On the inner product of modes

a) Email: melnola@elektro.dtu.dk

c) Current address: CSIRO Infrastructure Technologies, Private Bag 10, Clayton South, Victoria 3169, Australia 


\begin{abstract}
There are two definitions of the inner product of modal spatial functions used in the literature. Both definitions integrate the product of the modal spatial functions over a line, area, or volume. The only difference is that one of the definitions takes the complex conjugate of one of the modal spatial functions before multiplying the modes together. The definitions are the same if the modal spatial functions are real. If the modal spatial functions are complex, only the definition which takes the complex conjugate is an inner product. If the specific acoustic impedance of the boundaries has a real part, then the modes are only orthogonal with the definition which does not take the complex conjugate, although this definition is not strictly an inner product because the modal spatial functions are complex in this situation. However, this definition of "inner product" can be used to calculate the coefficients in the modal expansion of the system response. On the other hand, when it comes to calculating the mean pressure squared and the mean sound intensity, the modal spatial functions cross-products cannot be ignored because the modes are not orthogonal for the definition which takes the complex conjugate.
\end{abstract}

Keywords: inner product, orthogonality, modal theory, non-diffuse sound fields

PACS: 43.55.Br, 43.55.Cs, 43.55.Nd, 43.55.Ka 


\section{INTRODUCTION}

It is well-known that the statistical random-wave theory $\mathbf{1}$ is a good approximation to the sound field in a reverberant room in the frequency range where the modal overlap is high; that is, above the Schroeder frequency. ${ }^{2}$ In rooms where absorption is concentrated on certain surfaces (as in conventional measurements of sound absorption coefficients) ${ }^{3}$, the resulting sound field responds to non-uniform boundary conditions, and the conditions for the statistical theory are violated. To describe such sound field, a formal solution to the wave equation with the appropriate boundary conditions must be found, resulting in a description in terms of the modes of the room. Each mode corresponds to a frequency (the eigenfrequency or resonance frequency) and is solution to the homogeneous wave equation with the appropriate boundary conditions.

The modal theory of the stationary reverberant sound field has been studied extensively for rooms with nearly hard walls: Lyon $^{4}$ derived formulas for the spatial variance of the squared sound pressure and the input impedance of a pure-tone reverberant sound field assuming that the modal frequencies were locally spaced according to the Poisson or nearest-neighbor distributions; Davy ${ }^{5}$ corrected errors in Lyon's formulas; Weaver ${ }^{\mathbf{6}}$ pointed out that theoretically the Gaussian Orthogonal Ensemble (GOE) distribution should be used for the modal frequency spacings; Davy ${ }^{7}$ confirmed Weaver's results experimentally. Rooms with absorption concentrated on one of their walls have not received similar attention. Early derivations of the velocity potential for non-rigid walls are described by Morse and Bolt. ${ }^{8}$ The case of non-rigid walls is partially treated in Ref. 9, although the walls are assumed to have the same acoustic properties, thus excluding rooms with absorption concentrated on one of their walls. Kergomard et al. ${ }^{10}$ treat the one-dimensional case in great detail and give a number of references. They do allow the normalized specific acoustic impedances to be different at each end of the one-dimensional duct and show that the modal spatial functions are non-orthogonal if the normalized specific impedances have a non-zero real part indicating wall losses. 
Interestingly enough, the question of the orthogonality of the spatial modal functions in rectangular rooms with arbitrary boundary conditions has hardly been addressed. As a matter of fact, two different definitions of the inner product of modal spatial functions are used in the abovementioned literature. Both definitions integrate the product of the modal spatial functions over a line, area or volume; the only difference is that one of them takes the complex conjugate of one of the modal spatial functions before multiplying the modal spatial functions together. Essentially, they are the same if the modal spatial functions are real. These two definitions cause great confusion in the literature. For instance, Eq. (1) in a recent paper by Leader and $\operatorname{Pan}^{\mathbf{1 1}}$ on the modes of open cavities, gives the modal expansion formula for a closed cavity using the complex conjugate operator in both the numerator and the denominator while citing Eqs (9.4.1) and (9.4.2) of Ref. 12 which does not use the complex conjugate operator. Equations 7 to 10 of Ref. 11 also use the complex conjugate operator, while Herman ${ }^{\mathbf{1 3}}$ is cited for the orthogonality of the modal spatial functions of closed systems. However, Eq. (6.11) of Ref. 13 defines the inner product without using the complex conjugate operator. It appears that Herman ${ }^{\mathbf{1 3}}$ was assuming that his modal spatial functions were real because his "inner product" is not an inner product unless the modal spatial functions are real. [Footnote: To be fair to Leader and Pan, ${ }^{11}$ the authors would like to point out that the same error was made in a paper by Davy. ${ }^{5}$ ] If the modal spatial functions are complex, only the definition that takes the complex conjugate is an inner product. If the specific acoustic impedance of the boundaries has a real part (indicating wall losses), then the modes are only orthogonal with the definition that does not take the complex conjugate, although this definition is not strictly an inner product because the modal spatial functions are complex in this situation. However, this definition of "inner product" can be used to calculate the coefficients in the modal expansion of the room response. On the other hand, when it comes to calculating the mean pressure squared and the mean sound intensity, the modal spatial function cross-products cannot be ignored because the modes are not orthogonal for the definition 
which takes the complex conjugate. The subsequent extra-calculations is the reason why most authors prefer to assume (incorrectly) that the modal spatial functions are orthogonal with the (correct) definition of inner product which takes the complex conjugate of one of the modal spatial functions. The primary aim of this paper is to make researchers aware of these two definitions and to show that the modal spatial functions are orthogonal under the correct definition of inner product only if the real part of the specific acoustic impedance of the boundaries is null (that is, when the boundaries are free of absorption).

Further, because the boundary conditions depend on the wavenumber of the sound waves, these boundary conditions will vary with frequency even if the normalized specific acoustic impedances of the boundary are independent of frequency (the only exceptions - not considered in this paper - are the two extreme cases where the normalized specific acoustic impedance of the boundary is infinite or zero). Thus, the eigenfrequencies have to be calculated for each driving frequency of the sound source. There are multiple solutions to the equation for the eigen-frequencies of the modes, hence the selection of an adequate starting value for the numerical calculation is a crucial step. Starting the numerical solution with an analytical approximation to the eigenfrequencies works well at very low frequencies. At higher frequencies, the algorithm fails to converge to the correct solution. This is shown by a sudden jump in the calculated eigenfrequencies as a function of the exciting frequency. The paper shows that this problem can be overcome by means of a warmstart strategy, which implies that the result obtained for a given frequency is used as the starting point for the numerical solution of the eigenfrequency for the next higher driving frequency. For the lowest frequency at which calculations are made, the analytical approximation can be used as the starting point for the numerical solution. The paper also points out that a different analytic approximation is needed for the starting point of the numerical solution for the zeroth-order eigenfrequency at the lowest driving frequency. 
The paper is organized as follows: the mode model is presented in Section II. The orthogonality relation of the modes is investigated and a method of calculating suitable starting values for the numerical solution of the eigen-frequencies is given. In Section III, the derived model is used to examine properties of the sound pressure and complex sound intensity in a rectangular room with absorption concentrated on the floor. The numerical results are compared with theoretical predictions and a finite element model (FEM).

\section{THE MODE MODEL}

A three-dimensional rectangular room containing a highly absorptive surface and no scattering objects is the basis for the analysis in this study. The assumptions necessary for the calculations may be summarized as follows: $(i)$ The walls are assumed to be locally reacting, which means that their acoustic properties can be described by their respective normalized specific acoustic impedances " $\xi$ ”. Although the absorption shall be uniformly distributed on each wall, each of them may have different acoustic properties. No assumptions concerning the value of the normalized impedances are necessary; however, only walls with normalized impedances larger than unity are considered in this paper. For convenience, we will also consider real values of the normalized impedances (although the model is not limited to real values). The assumption that $\xi$ is real is not very restrictive, since an imaginary part simply displaces the resonant frequencies by a small amount corresponding to a small change of room dimensions; ${ }^{14}$ (ii) The analysis is confined to pure-tone sound fields, although some pure-tone results are averaged to obtain third-octave band results, and only stationary sound fields (and, accordingly, only time-averaged quantities) are considered; (iii) The sound field is produced by a single monopole source with infinite acoustic impedance; (iv) Air absorption is assumed to be negligible.

The sound field produced by a monopole point source of volume velocity $Q$ oscillating at angular frequency $\omega$ in a rectangular parallelepiped room of dimensions $L_{x}, L_{y}$ and $L_{z}$, is calculated 
using the well-known modal expansion method. ${ }^{\mathbf{1 2}}$ The steady-state pressure response as a function of position is a solution to the wave equation

$$
\nabla^{2} p(\mathbf{x})+k^{2} p(\mathbf{x})=-i \omega \rho Q \delta\left(\mathbf{x}-\mathbf{x}_{0}\right)
$$

where $\mathbf{x}=(x, y, z)$ and $\mathbf{x}_{0}=\left(x_{0}, y_{0}, z_{0}\right)$ are the spatial coordinates of the receiver and source, respectively, $\rho$ is the air density and $\delta\left(\mathbf{x}-\mathbf{x}_{0}\right)$ is the three-dimensional Dirac delta function. The time dependency $e^{i \omega t}$ is disregarded. The solution to Eq. (1) can be expressed in terms of the modal functions of the room if these modal spatial functions form a complete basis for the space of solutions satisfying the given boundary conditions; i.e.,

$$
p(\boldsymbol{x})=\sum_{n} A_{n} p_{n}(\boldsymbol{x})
$$

and

$$
q(\boldsymbol{x})=Q \delta\left(\boldsymbol{x}-\boldsymbol{x}_{\mathbf{0}}\right)=\sum_{n} B_{n} p_{n}(\boldsymbol{x})
$$

where $p_{n}$ is the $n$th modal spatial function. The coefficients $A_{n}$ and $B_{n}$ can be easily determined if the modal spatial functions are orthogonal for a suitable definition of inner product. The modal functions $p_{n}$ are solutions to the homogeneous wave equation

$$
\nabla^{2} p_{n}(\mathbf{x})+k_{n}^{2} p_{n}(\mathbf{x})=0
$$

with the boundary conditions

$$
\xi \frac{\partial p_{n}(\mathbf{x})}{\partial N}=-i k p_{n}(\mathbf{x})
$$

where $\xi$ is the normalized specific acoustic impedance and $\partial / \partial N$ denotes partial differentiation in the direction of the normal to the surface, pointing outward. Equation (3) has solutions only for certain discrete complex values of $k$, the modal frequencies (or eigenvalues) $k_{n}$.

\section{II.A Modal frequencies and modal functions}


To apply the modal decomposition given in Eqs (2a) and (2b), one should first solve for the eigenvalues and eigenfunctions of Eq. (3) with the boundary conditions defined in Eq. (4). The calculation details are given in the one-dimensional case and extended to three dimensions using separation of variables. The one-dimensional medium extends from $x=0$ to $x=L_{x}$, and a source of volume velocity per unit area $Q_{S}$ is placed at $x=x_{0}$. The sound pressure in the one-dimensional case is solution of the inhomogeneous equation $d^{2} p(x) / d x^{2}+k^{2} p(x)=-i \omega \rho Q_{S} \delta\left(x-x_{0}\right)$, and the onedimensional modal functions in the $x$-direction can conveniently be written as

$$
p_{n}(x)=C_{n} e^{-i k_{n_{x}} x}+D_{n} e^{i k_{n_{x}} x}
$$

By inserting Eq. (5) into the boundary conditions defined in Eq. (4), a system of two linear homogeneous equations for the constants $C_{n}$ and $D_{n}$ is obtained:

$$
\left\{\begin{array}{c}
-C_{n}\left(k_{n_{x}} \xi_{x, 0}+k\right)+D_{n}\left(k_{n_{x}} \xi_{x, 0}-k\right)=0 \\
C_{n}\left(-k_{n_{x}} \xi_{x, L_{x}}+k\right) e^{-i k_{n_{x}} L_{x}}+D_{n}\left(k_{n_{x}} \xi_{x, L_{x}}+k\right) e^{i k_{n_{x}} L_{x}=0}
\end{array}\right.
$$

where $\xi_{x, 0}$ denotes the normalized impedance of the wall at $x=0$, and $\xi_{x, L_{x}}$ that of the wall at $x=L_{x}$. This system of equations has a non-vanishing solution only if the determinant of their coefficients is zero, which leads to the following condition:

$$
e^{2 i k_{n_{x}} L_{x}}=\frac{\left(k_{n_{x}} \xi_{x, 0}-k\right)\left(k_{n_{x}} \xi_{x, L_{x}}-k\right)}{\left(k_{n_{x}} \xi_{x, 0}+k\right)\left(k_{n_{x}} \xi_{x, L_{x}}+k\right)}
$$

Solving Eq. (7) for $k_{n_{x}} L_{x}$ yields the transcendent equation

$$
\tan \left(k_{n_{x}} L_{x}\right)=\frac{i k L_{x}\left(\xi_{x, 0}+\xi_{x, L_{x}}\right)}{k_{n_{x}} L_{x}\left(\xi_{x, 0} \xi_{x, L_{x}}+\frac{\left(k L_{x}\right)^{2}}{\left(k_{n_{x}} L_{x}\right)^{2}}\right)}
$$

which must be solved numerically to determine the modal frequencies $k_{n_{x}}$ (details concerning the numerical resolution will be given in section II.D). Analogous equations can be derived in the $y$ - and $z$-directions. Using separation of variables, the three-dimensional modal functions may take the form 
$p_{n}(\mathbf{x})=\prod_{j=x, y, z} \cosh \left(i k_{n_{j}} j+\phi_{n_{j}}\right)$, where the phase terms $\phi_{n_{j}}$ are derived from the boundary conditions and given in Eq. (18) [one can also use the equivalent complex exponential formulation as in Eq. (5)]. The allowed values of $k_{n_{x}}, k_{n_{y}}$ and $k_{n_{z}}$ are calculated separately using Eq. (8) [and its equivalents in the $y$ - and $z$-directions], and determine the value of $k_{n}^{2}=k_{n_{x}}^{2}+k_{n_{y}}^{2}+k_{n_{z}}^{2}$.

\section{II.B Orthogonality relations of the room modes and completeness}

This section derives the orthogonality relations for the modal spatial functions commonly used to calculate the coefficients $A_{n}$ and $B_{n}$ in Eqs. (2a) and (2b).

\section{II.B.1 Classic inner product}

First, the correct inner product for complex-valued functions of real variables

$$
\iiint_{V} p_{n}(\mathbf{x}) p_{m}^{*}(\mathbf{x}) d V
$$

where $*$ denotes the complex conjugate, is considered. Calculation details are given for the onedimensional medium defined in Section II. A. The extension to the three-dimensional case is straightforward. The one-dimensional eigenfunctions $p_{n}(x)$ and $p_{m}(x)$ satisfy Eq. (3) with the boundary condition given in Eq. (4). Analogous equations for $p_{m}^{*}(x)$ are obtained by taking the complex conjugate on both sides of Eqs (3) and (4). Integrating the product $p_{n}(x) p_{m}^{*}(x)$ over the onedimensional domain yields

$$
\left(\left(k_{m_{x}}^{2}\right)^{*}-k_{n_{x}}^{2}\right) \int_{0}^{L_{x}} p_{n}(x) p_{m}^{*}(x) d x=\int_{0}^{L_{x}}\left(\frac{d^{2} p_{n}(x)}{d x^{2}} p_{m}^{*}(x)-\frac{d^{2} p_{m}^{*}(x)}{d x^{2}} p_{n}(x)\right) d x
$$

Using integration by parts on the right-hand side and inserting the boundary condition defined in Eq.

$$
\int_{0}^{L_{x}} p_{n}(x) p_{m}^{*}(x) d x
$$




$$
=\frac{-i k}{\left(\left(k_{m_{x}}^{2}\right)^{*}-k_{n_{x}}^{2}\right)}\left(\left(\frac{1}{\xi_{x, L_{x}}}+\frac{1}{\left(\xi_{x, L_{x}}\right)^{*}}\right) p_{n}\left(L_{x}\right) p_{m}^{*}\left(L_{x}\right)+\left(\frac{1}{\xi_{x, 0}}+\frac{1}{\left(\xi_{x, 0}\right)^{*}}\right) p_{n}(0) p_{m}^{*}(0)\right) \text {. }
$$

Equation (11) can be extended to the three-dimensional case using separation of variables, yielding

$$
\begin{aligned}
& \iiint_{V} p_{n}(\mathbf{x}) p_{m}^{*}(\mathbf{x}) d V= \\
& \mathrm{i} \prod_{j=x, y, z} \frac{k}{\left(\left(k_{m_{j}}^{2}\right)^{*}-k_{n_{j}}^{2}\right)}\left(\left(\frac{1}{\xi_{j, L_{j}}}+\frac{1}{\left(\xi_{j, L_{j}}\right)^{*}}\right) p_{n_{j}}\left(L_{j}\right) p_{m_{j}}^{*}\left(L_{j}\right)+\left(\frac{1}{\xi_{j, 0}}+\frac{1}{\left(\xi_{j, 0}\right)^{*}}\right) p_{n_{j}}(0) p_{m_{j}}^{*}(0)\right) .
\end{aligned}
$$

It is apparent from Eq. (12) that the scalar product defined in Eq. (9) does not equal zero, except when the walls are free of absorption. This would be the case if the walls are rigid $(\xi=\infty)$, or more generally if they are purely reflective (then their normalized impedances are purely imaginary). Whenever the normalized impedances have a non-zero real part indicating wall losses, the modes are found to be non-orthogonal for the scalar product defined in Eq. (9).

\section{II.B.2 Modified "inner product"}

In the following, it is shown that the modified "inner product"

$$
\iiint_{V} p_{n}(\mathbf{x}) p_{m}(\mathbf{x}) d V
$$

leads to the orthogonality of the modes. Following a similar derivation as for Eq. (11), gives

$$
\begin{aligned}
& \int_{0}^{L_{x}} p_{n}(x) p_{m}(x) d x \\
& =\frac{1}{\left(k_{m_{x}}^{2}-k_{n_{x}}^{2}\right)}\left(-\frac{i k}{\xi_{x, L_{x}}} p_{m}\left(L_{x}\right) p_{n}\left(L_{x}\right)+\frac{i k}{\xi_{x, L_{x}}} p_{n}\left(L_{x}\right) p_{m}\left(L_{x}\right)-\frac{i k}{\xi_{x, 0}} p_{m}(0) p_{n}(0)+\frac{i k}{\xi_{x, 0}} p_{n}(0) p_{m}(0)\right),
\end{aligned}
$$

which equals zero for any value of the normalized impedances provided that $m \neq n$. Note that freely decaying modes are not necessarily orthogonal because the value of the normalized wall impedances is a function of the eigenfrequency of each mode. The driven modes are orthogonal because the 
normalized wall impedance in this case is a function of the driving frequency. Further, a relation for $m=n$ can be found by calculating the product $\int_{0}^{L_{x}} p_{n}^{2}(x) d x$. For this purpose, it is convenient to write the modal functions in the form $p_{n}(x)=\cosh \left(i k_{n_{x}} x+\phi_{n_{x}}\right)$, which is equivalent to the exponential form in Eq. (5):

$$
\int_{0}^{L_{x}} p_{n}^{2}(x) d x=\frac{L_{x}}{2}\left(1+\frac{1}{i k_{n_{x}} L_{x}} \sinh \left(i k_{n_{x}} L_{x}\right) \cosh \left(i k_{n_{x}} L_{x}+2 \phi_{n_{x}}\right)\right),
$$

where use is made of the change of variable $u=i k_{n_{x}} x+\phi_{n_{x}}$. The value of $\phi_{n_{x}}$ can be determined from the boundary condition $\xi_{x, 0} d p_{n}(0) / d x-i k p_{n}(0)=0$, which gives $\phi_{n_{x}}=\operatorname{coth}^{-1}\left(\xi_{x, 0} k_{n_{x}} / k\right)$. Using separation of variables, the foregoing derivation leads to the orthogonality of the modes in the threedimensional case:

$$
\iiint_{V} p_{n}(\mathbf{x}) p_{m}(\mathbf{x}) d V= \begin{cases}K_{n} & n=m \\ 0 & n \neq m\end{cases}
$$

with

$$
K_{n}=\prod_{j=x, y, z} \frac{L_{j}}{2}\left(1+\frac{1}{i k_{n_{j}} L_{j}} \sinh \left(i k_{n_{j}} L_{j}\right) \cosh \left(i k_{n_{j}} L_{j}+2 \phi_{n_{j}}\right)\right)
$$

and

$$
\phi_{n_{j}=x, y, z}=\operatorname{coth}^{-1}\left(\xi_{j, 0} k_{n_{j}} / k\right) .
$$

This shows that the modal functions are orthogonal for the "inner product" defined in Eq. (13). However, the complex-valued modal functions are not orthogonal in the strict sense, as this would require that the product defined in Eq. (13) is an inner product on the space of possible solutions that satisfy the boundary conditions defined in Eq. (4). However, the product defined in Eq. (13) does not satisfy the definition ${ }^{15}$ of an inner product on the aforementioned space. Nevertheless, the orthogonality relation derived in Eqs (16) to (18) is sufficient for solving the wave equation, but not 
sufficient to eliminate the need to evaluate the average values over the room space of cross-products of modal spatial functions. Besides, it is worth mentioning that for rigid walls, the calculation of the quantity defined in Eq. (13) is equivalent to the calculation of the quantity defined when replacing $p_{m}$ by its conjugate. It follows that the modal functions (in this case real-valued) are strictly orthogonal. This property has been extensively used in the literature. ${ }^{4-9}$

\section{II.B.3 Completeness}

The completeness of the real-valued modal functions in the case of rigid walls is known. ${ }^{4-9}$ The question of the completeness of the complex-valued modal functions for the set of possible solutions satisfying the boundary conditions defined in Eq. (4) is outside the scope of this investigation. The solution to Eq. (1) obtained under the assumption of completeness of the modal functions and the orthogonality relation derived in Eqs (16) to (18) shows perfect agreement with the simple closedform solution in the one-dimensional case ${ }^{\mathbf{1 0}}$ (not shown for conciseness). This means that the complex-valued modal functions must be close to being a complete basis of elements, which becomes orthogonal for the product defined in Eq. (13). As a consequence, we may say that the definition of orthogonality proposed in Eq. (13) enables the rigorous solution of the wave equation if the modes form a complete basis for the set of possible solutions satisfying the boundary conditions defined in Eq. (4).

\section{II.C Steady-state response}

In the following, as discussed above, it is assumed that the complex-valued modal functions for the set of possible solutions satisfying the boundary conditions defined in Eq. (4) is complete. Once the modal spatial functions and modal frequencies have been determined, one may expand the solution to Eq. (1) in terms of the modal functions $p_{n}$ as shown in Eq. (2a). The orthogonality of the modes, necessary to calculate the coefficients $A_{n}$ and $B_{n}$, is derived in section II.B, where it is shown that the modes are not orthogonal with the usual definition of inner product if the normalized wall specific 
acoustic impedances have a non-zero real part [see Eq. (12)]. A modified definition of orthogonality is necessary [see Eqs (16) to (18)], which gives

$$
B_{n}=\frac{1}{K_{n}} \iiint_{V} q(\mathbf{x}) p_{n}(\mathbf{x}) d V=\frac{Q p_{n}\left(\mathbf{x}_{0}\right)}{K_{n}} .
$$

Expanding both sides of Eq. (1) in terms of the modal functions and combining with Eqs (3) and (19), we have

$$
A_{n}=\frac{i \omega \rho B_{n}}{\left(k_{n}^{2}-k^{2}\right)}=\frac{i \omega \rho Q p_{n}\left(\mathbf{x}_{0}\right)}{K_{n}\left(k_{n}^{2}-k^{2}\right)}
$$

and, using the expansion defined in Eq. (2a)

$$
p(\boldsymbol{x})=\sum_{n} \frac{i \omega \rho Q p_{n}\left(\boldsymbol{x}_{0}\right) p_{n}(\boldsymbol{x})}{K_{n}\left(k_{n}^{2}-k^{2}\right)}
$$

where $k_{n}^{2}$ satisfies the condition given in Eq. (10). The particle velocity components can now be derived from Euler's equation of motion:

$$
u_{j=x, y, z}(\mathbf{x})=-Q \sum_{n} \frac{p_{n}\left(\mathbf{x}_{0}\right) \frac{\partial p_{n}(\mathbf{x})}{\partial j}}{K_{n}\left(k_{n}^{2}-k^{2}\right)}
$$

and the active and reactive intensity vectors are calculated as

$$
\begin{aligned}
& \mathbf{I}(\mathbf{x})=\operatorname{Re}\left\{p(\mathbf{x}) \mathbf{u}^{*}(\mathbf{x})\right\} \\
& \mathbf{J}(\mathbf{x})=\operatorname{Im}\left\{p(\mathbf{x}) \mathbf{u}^{*}(\mathbf{x})\right\}
\end{aligned}
$$

It can be remarked that to calculate the sound pressure or the real and imaginary parts of the sound intensity at a position in the room, the modified definition of "inner product" given by Eq. (13) needs to be used. However, to calculate the mean squared sound pressure or the real and imaginary parts of the sound intensity averaged across the whole of the room volume using $N$ modes, it is necessary to evaluate $N^{2}$ integrals of the form of Eq. (9). If the modes were orthogonal for the definition of inner product given by Eq. (9), all but the $N$ diagonal products would be zero, which would substantially reduce the amount of calculation. 
In addition, a theoretical expression for the spatial standard deviation of the sound pressure level of third-octave bands of random noise in a rectangular room with arbitrary boundary conditions is given in the Appendix. The theoretical expression is based on the theory presented by Davy. ${ }^{\mathbf{1 6}}$ An expression for the damping rate $\gamma_{n}$ of the $n$th modal amplitude which is used in the theoretical expressions is derived in the Appendix for the case of arbitrary boundary conditions. The modal damping rate is a term that appears in the expansion of the $n$th modal wavenumber $k_{n}=\left(\omega_{n}+i \gamma_{n}\right) / c$, where the resonant angular frequency of the $n$th mode is $\omega_{n}$.

\section{II.D Numerical solution}

The solution of Eq. (8) is computed for each of the three axes using the trust-region ${ }^{\mathbf{1 7}}$ fsolve algorithm, available under the Optimization Toolbox in the Matlab environment (MathWorks, 2018). ${ }^{\mathbf{1 8}}$ The requirement of an initial guess as a starting point is a critical step. In this section, the determination of an adequate approximate starting value for the numerical calculation of the modal frequencies $k_{n_{i}}$ for $i=x, y$ and $z$ is addressed.

Considering a one-dimensional medium with rigid or nearly hard terminations (i.e., $\left|\xi_{x, 0}\right| \gg$ 1 and $\left|\xi_{x, L_{x}}\right| \gg 1$ ), an approximate solution to Eq. (8) can be found by applying the series expansion of the exponential function truncated after the second term to the right-hand side of Eq. (7):

$$
e^{2 i k_{n_{x}} L_{x}}=\frac{\left(1-\frac{k}{k_{n_{x}} \xi_{x, 0}}\right)\left(1-\frac{k}{k_{n_{x}} \xi_{x, L_{x}}}\right)}{\left(1+\frac{k}{k_{n_{x}} \xi_{x, 0}}\right)\left(1+\frac{k}{k_{n_{x}} \xi_{x, L_{x}}}\right)} \approx e^{\frac{-2 k}{k_{n_{x}}}\left(\frac{1}{\xi_{x, 0}}+\frac{1}{\xi_{x, L_{x}}}\right)}
$$

Since the exponential function with imaginary argument is $2 \pi$-periodic, $e^{2 i k_{n_{x}} L_{x}}=e^{2 i k_{n_{x}} L_{x}+2 i n \pi} \approx$ $e^{\frac{-2 k}{k_{n_{x}}}}\left(\frac{1}{\xi_{x, 0}}+\frac{1}{\xi_{x, L_{x}}}\right)$, where $n$ is an arbitrary integer. Equating the exponents yields

$$
k_{n_{x}} L_{x} \approx n \pi+\frac{i k}{k_{n_{x}}}\left(\frac{1}{\xi_{x, 0}}+\frac{1}{\xi_{x, L_{x}}}\right) .
$$


Since $\left|\xi_{x, 0}\right| \gg 1$ and $\left|\xi_{x, L_{x}}\right| \gg 1$, the second term on the right-hand side is much smaller than the first one, so that we may replace $k_{n_{x}}$ in the denominator by its expression in the hard-wall case $\left(n \pi / L_{x}\right)$, which gives

$$
k_{n_{x}} \approx \frac{n \pi}{L_{x}}+\frac{i k}{n \pi}\left(\frac{1}{\xi_{x, 0}}+\frac{1}{\xi_{x, L_{x}}}\right) .
$$

It is worth mentioning that in the case of rigid or nearly rigid terminations, the well-known expression $k_{n_{x}}=n \pi / L_{x}$ is found. Besides, the modal frequencies are complex unless the terminations are absorption free. For infinite or purely imaginary normalized impedances, the modal frequencies are real. This corresponds to the case where the boundaries are rigid or purely reflective, respectively. In the latter case, the modal frequencies will simply be slightly shifted compared to the rigid termination case.

The frequency-dependent approximate solution given in Eq. (26) is a tempting initial point for the solution of Eq. (8). The algorithm however sometimes fails to converge to the correct solution when using the approximate solution as an initial guess for the solution of Eq. (8). This is shown by a sudden jump in the modal frequency for a given mode as a function of exciting frequency. Therefore, a warm-start strategy is used instead, which implies that the result obtained for a given frequency is used as the starting point for the calculation of the eigenfrequency for the next higher driving frequency. The approximate solution given in Eq. (26) is used for the computation at $1 \mathrm{~Hz}$.

For each frequency, the zeroth-order modal frequency must be calculated separately, as it is apparent that Eq. (26) is not valid for the zeroth-order mode where $n=0$. An alternative approximate value for $k_{0, x}$ can be found by using the series expansion of the tangent function truncated after the first term. Since $k_{0, x} L_{x} \ll 1$, this expansion can be applied to the left-hand side of Eq. (8), which yields 


$$
k_{0, x} \approx \frac{1}{L_{x}} \sqrt{-\frac{\left(k L_{x}\right)^{2}}{\xi_{x, 0} \xi_{x, L_{x}}}+i k L_{x}\left(\frac{1}{\xi_{x, 0}}+\frac{1}{\xi_{x, L_{x}}}\right)}
$$

In the particular case of a rectangular room with absorption concentrated on the floor, and two pairs of opposite walls which are rigid or nearly rigid, one may use the approximate solutions defined in Eqs (26) and (27) in the corresponding horizontal directions. In this study, however, the numerically exact values in all directions were always calculated. Also note that the cases when the real part of the normalized impedance is less than unity or when the imaginary part is very different from zero are not dealt with in this paper. These two cases probably need different starting values for the eigenfrequencies, the assumption of nearly-rigid walls used in Eqs (24) to (27) will become less correct.

\section{NUMERICAL RESULTS}

The mode model described in Section II is used to examine properties of the sound pressure and complex sound intensity in an existing small $\left(40 \mathrm{~m}^{3}\right)$ rectangular room with dimensions $4.38 \times 3.29$ x $2.97 \mathrm{~m}$. A point source is placed in the upper corner at $\left(0.6 ; 0.5 ; L_{z}-0.4\right) \mathrm{m}$ (that is, at least $0.4 \mathrm{~m}$ from any boundary). This position was chosen after calculations with a point source, placed closer to the corner and spaced at the equal distance of $0.25 \mathrm{~m}$ from each of the three walls forming the corner, unsurprisingly produced atypical results. Two configurations of the room are considered: $(i)$ all surfaces are nearly rigid (i.e., $|\xi| \gg 1$ ); (ii) the floor is covered with absorptive material of varying absorption while the remaining surfaces are nearly rigid. For simplicity, the normalized impedances have the same values across frequency, although this is not necessary for the calculation method. The pressure, particle velocity and complex intensity fields are calculated at 378 points, over a regular grid of receivers placed $0.4 \mathrm{~m}$ away from any wall. Receivers closer than $1 \mathrm{~m}$ to the source are not used. The steady-state pressure response is calculated according to Eq. (21). The allowed values of 
$k_{n_{x}}, k_{n_{y}}$ and $k_{n_{z}}$ are calculated separately using Eq. (8). The algorithm described in Section II.D is carried out from $1 \mathrm{~Hz}$ to $450 \mathrm{~Hz}$ with a frequency step of $1 \mathrm{~Hz}$.

Figure 1 shows the computed sound pressure level in the undamped (left) and damped (right) room (in this case the floor is covered with absorptive material with specific acoustic impedance $\xi_{z}=0$ $=2$, corresponding to an absorption coefficient $\alpha_{z=0}=0.9$ ). The results are displayed at $70 \mathrm{~Hz}, \mathrm{a}$ natural frequency corresponding to the $(1,0,1)$ mode (this natural frequency is estimated in the undamped case). The results (interpolated for clarity) are displayed as orthogonal slice planes normal to the $x$-, $y$-, and $z$-direction, respectively. The mode is clearly identified in the results from the undamped room (left). Yet, in the damped room (right), wave motion solely occurs in the plane parallel to the absorbing surface. This is in agreement with the general behavior of sound propagating in a rectangular room containing a highly absorptive surface and no scattering objects, as described in e.g., Refs 19 and 20: the non-grazing part of the sound field is greatly damped by the absorbing surface, while the grazing part (propagating parallel to the absorbing wall) is much less affected by the absorption.

Additionally in Figure 1, the sound pressure level computed from the analytical model described in Section II is systematically compared with finite element model (FEM) calculations. All calculations were made using the FEM software package ${ }^{21}$ COMSOL 5.3a, where the longuest dimension of the elements $h$ was adjusted to guarantee that $k h / p<1$ ( $p=2$ is the element order). ${ }^{\mathbf{2 2}}$ There is fair, if not perfect, agreement between the FEM and analytical results. The spatially averaged relative difference $\langle\varepsilon\rangle[\%]=100\left\|\mathbf{x}-\mathbf{x}_{\mathrm{FEM}}\right\|_{2} /\|\mathbf{x}\|_{2}$ (where the vector $\mathbf{x}$ corresponds to the analytical sound pressure, $\mathbf{x}_{\mathrm{FEM}}$ to the FEM model values, and $\|.\|_{2}$ indicates the $l_{2}$-norm of the pressure vectors) yields values ranging between 4 and $6 \%$ in the entire frequency range (mean values for third-octave bands). This indicates that the proposed numerical strategy for solving Eq. (8) is robust. 
The fluctuations in sound pressure level across the volume of the room as a function of frequency are shown in Figure 2. The spatial standard deviation of the sound pressure level [in $\mathrm{dB}$ ] with respect to the 378 receiver positions is computed using the analytical model described in Section II and validated against the theoretical predictions presented in the Appendix. The results are displayed as mean values per third-octave band (ranging from $50 \mathrm{~Hz}$ to $400 \mathrm{~Hz}$ ) for three different absorptions of the floor surface $\left(\alpha_{z=0}=0.9, \alpha_{z=0}=0.3\right.$ and rigid floor, respectively). There is fair agreement between numerical and theoretical predictions. It is confirmed that the spatial standard deviation of the sound pressure level decreases with increasing frequency as the modal overlap increases and exhibits lower values in the undamped case at high frequencies. In fact, the narrower modal bandwidth in the undamped case means that there are more modal frequencies in each thirdoctave band at which the sound pressure is uncorrelated with the sound pressure at the other modal frequencies. The smaller modal overlap produces the opposite result at low frequencies. The increase of the modal overlap and the third-octave bandwidth with frequency is responsible for the decrease as the frequency increases. At low frequencies, the computed spatial standard deviation fluctuates with frequency. This is expected since the spatial standard deviation at low modal overlap depends strongly on whether one single mode or several modes contribute to the sound field. The damped case has a smaller standard deviation because of the larger modal overlap. At higher frequencies, the opposite occurs because the wider modal bandwidth means less statistically independent modal frequencies in each third-octave band.

Figure 3 shows a volumetric representation of the active intensity field in the undamped and damped room $\left(\alpha_{z=0}=0.9\right)$, respectively, for the third-octave band centered at $125 \mathrm{~Hz}$. The intensity vectors are computed at the 378 receiver points, although positions closer than $1 \mathrm{~m}$ from the source are not used. The length of the vectors is proportional to the intensity (in $\left[\mathrm{W} / \mathrm{m}^{2}\right]$ ) at the base of the cones, and the cones are color-coded to also show the magnitude of the intensity vector. For both 
damping conditions, it is apparent that the intensity field emanates from the direction of the source placed at $\left(0.6 ; 0.5 ; L_{z}-0.4\right) \mathrm{m}$ (black dot in Fig. 3). In the undamped room, the intensity field seems to flow in multiple directions. When absorption is added to the floor, the magnitude of the active intensity is less, and the flow is pointing towards the absorbing surface (curving from the source to the absorbing surface). This is in agreement with previous findings. ${ }^{23}$

Figure 4 shows a volumetric representation of the active (top) and reactive (bottom) intensity fields at $58 \mathrm{~Hz}$, a natural frequency corresponding to the $(0,0,1)$ mode (natural frequency estimated in the rigid case). The intensity fields are represented in the undamped room (left) and the damped room (right), respectively. In the undamped room, the magnitude of the active intensity is about onethird of that of the reactive intensity. It is apparent that the sound field is strongly reactive, as indicated by the presence of a standing wave in the $z$-direction. These results agree with theoretical predictions [24-27]. In the damped room, the magnitudes of the active and reactive intensities are lower than in the undamped case. The active intensity is clearly pointing towards the absorbing surface, although slightly angled in the $x$ - and $y$-directions, corresponding to the position of the source. The magnitude of the active intensity field is also larger than that of the reactive intensity, indicating a more dominant active intensity flow, as expected due to the presence of the absorbing floor. It is also noticeable that the reactive flows occur only in the $x y$-plane (plane parallel to the absorbing surface), indicating a lack of a standing wave between the floor and the ceiling.

Figure 5 compares the spatial average of the active and reactive intensity magnitudes in the undamped [Fig. 5(a)] and damped [Fig. 5(b)] room as a function of frequency (the regions of low and high modal overlap are represented separately). The modes are clearly identified in the undamped room at low modal overlap. The sound field is strongly reactive in all modes, in agreement with the theoretical considerations presented in Ref. 27. The modal overlap is higher in the damped room, and the magnitude of the active intensity field is larger than that of the reactive intensity, as expected due 
to the presence of the absorbing floor. At higher modal overlap in the undamped room, the sound field remains strongly reactive in all modes, and active and reactive intensities are of comparable magnitude. In the damped room, it is noticeable that oblique modes are affected by the presence of the absorbing floor (the reactive intensity at the corresponding frequencies is lower than in the undamped room), while this is not necessarily the case for tangential and axial modes, depending on their orientation with respect to the absorbing surface.

\section{CONCLUSION}

A mode model has been used to examine the steady-state sound field in a rectangular room with sound absorption concentrated on the floor. The existing modal theory for the reverberant sound field in rectangular rooms with nearly hard walls has been extended to the case of rectangular rooms whose walls have normalized specific acoustic impedances which can differ between walls, and the question of the orthogonality of the spatial modal functions in this case has been examined. The mathematical derivations exhibit interesting properties of the modes: in particular, it is shown that the spatial modal functions are not necessarily orthogonal with the usual definition of inner product, which leads to a modified definition of orthogonality. This modified definition enables the calculation of the modal expansion coefficients but does not guarantee that the average of the cross-products of modal spatial functions are zero when averaged over the room volume.

A method for finding starting values for the numerical calculation of the eigenfrequencies has been proposed, which guarantees that the numerical algorithm converges to the correct solution. A different approximate starting value for the numerical calculation of the eigen-frequency of the zeroth-order mode at the lowest frequency is also given. These numerical considerations have been confirmed with sound pressure calculations using a finite element model (FEM).

The model makes it possible to analytically derive expressions characterizing the sound pressure and the intensity flows corresponding to varying positions throughout the volume of the 
room. A theoretical expression for the spatial standard deviation of the sound pressure level in rooms with arbitrary boundary conditions has been presented. Good agreement was found between this theoretical prediction and a direct computation of the spatial fluctuations of the sound pressure level using the modal expansion method given in this paper. The properties of the active and reactive intensity flows have been examined and found to compare well with the predicted behaviours in the literature. The model provides a powerful means to accurately describe fundamental properties (e.g., sound pressure, particle velocity, complex intensity) of rooms with non-diffuse sound fields and, as such, may find application in the study of e.g., reverberation chambers used for conventional measurements of sound absorption, or ordinary rooms (classrooms, clinical rooms, etc.).

\section{ACKNOWLEDGMENTS}

This work is funded by the Oticon Foundation. The authors would like to thank Peter Risby Andersen for advice with the FEM simulations.

\section{APPENDIX: SPATIAL STANDARD DEVIATION OF THE SOUND PRESSURE LEVEL IN A ROOM WITH ARBITRARY BOUNDARY CONDITIONS}

This appendix shows the derivation of the theoretical expression for the spatial standard deviation of the sound pressure level. The theoretical considerations in Ref. [16] are extended to the case of a room with arbitrary boundary conditions. An expression for the damping rate of the modes is derived. In Section III, the derived expression is compared with the computed values.

The theoretical spatial standard deviation $s$ of the sound pressure level in a reverberant space excited by a frequency band of random noise is given by ${ }^{16}$

$$
s=\sqrt{\left(10 \log _{10} e\right)^{2} r},
$$

with ${ }^{16}$ 


$$
r=\frac{1}{Z}\left(1+\frac{K}{M_{s}}\right) F(\pi Z)
$$

and $Z=B_{s f} / \gamma$, where $B_{s f}$ is the statistical bandwidth in $\mathrm{Hz}$, and $\gamma$ is the damping rate of the modal amplitudes. $M_{S}=n \gamma$ is the statistical modal overlap, which is the product of the modal density $n$ with the statistical modal bandwidth (here in $\mathrm{Hz}$ ). The function $F$ reads ${ }^{\mathbf{1 6}}$

$$
F(\pi Z)=\frac{2}{\pi} \arctan (\pi Z)-\frac{1}{\pi^{2} Z} \ln \left(1+(\pi Z)^{2}\right)
$$

and

$$
K=\left(\frac{\left\langle p_{n}^{4}(\boldsymbol{x})\right\rangle}{\left\langle p_{n}^{2}(\boldsymbol{x})\right\rangle^{2}}\right)^{2}-3 C\left(M_{s}\right)
$$

where $p_{n}(\mathbf{x})$ is a modal spatial function, and the angular brackets $\langle\cdot\rangle$ denote the average value over position in the room and over modes in the frequency range of interest. $C$ is a function of the distribution of the modal frequency spacings and reads ${ }^{\mathbf{1 6}}$

$$
\begin{aligned}
& 3 C\left(M_{s}\right)=\left(\frac{M_{s}}{4}+2-\frac{5}{4 M_{s}}\right)+e^{-2 M_{s}}\left(\frac{M_{s}}{4}+\frac{1}{2}+\frac{5}{4 M_{s}}\right)-E_{1}\left(M_{s}\right) e^{-M_{s}}\left(\frac{M_{s}^{2}}{4}+\frac{3 M_{s}}{4}+\frac{5}{2}+\frac{5}{2 M_{s}}\right)- \\
& E_{1}\left(M_{s}\right) e^{M_{s}}\left(\frac{M_{s}^{2}}{4}-\frac{3 M_{s}}{4}+\frac{5}{2}-\frac{5}{2 M_{s}}\right),
\end{aligned}
$$

where $E_{1}$ is the exponential integral. The modal density $n$ may be calculated using the formulas for a rectangular parallelepiped room with rigid walls ${ }^{5}$ and reads

$$
n=\frac{4 \pi f^{2} L_{x} L_{y} L_{z}}{c^{3}}+\frac{\pi f}{c^{2}}\left(L_{x} L_{y}+L_{x} L_{z}+L_{y} L_{z}\right)+\frac{1}{2 c}\left(L_{x}+L_{y}+L_{z}\right) .
$$

Additionally, for all modes $\left\langle p_{n}^{2}(\mathbf{x})\right\rangle=1$, and $\left\langle p_{n}^{4}(\mathbf{x})\right\rangle=(3 / 2)^{3},\left\langle p_{n}^{4}(\mathbf{x})\right\rangle=(3 / 2)^{2},\left\langle p_{n}^{4}(\mathbf{x})\right\rangle=3 / 2$ for oblique, tangential and axial modes, respectively. 5 Thus

$$
\frac{\left\langle p_{n}^{4}(\mathbf{x})\right\rangle}{\left\langle p_{n}^{2}(\mathbf{x})\right\rangle^{2}}=\frac{1}{n}\left(\frac{27}{8} \frac{4 \pi f^{2} L_{x} L_{y} L_{z}}{c^{3}}+\frac{9}{8} \frac{\pi f}{c^{2}}\left(L_{x} L_{y}+L_{x} L_{z}+L_{y} L_{z}\right)+\frac{3}{8} \frac{1}{2 c}\left(L_{x}+L_{y}+L_{z}\right)\right) .
$$


In order to estimate the modal damping rate $\gamma$, the square of the wave number is expressed in the form

$$
k_{n}^{2}=\sum_{j=x, y, z} k_{n_{j}}^{2}=\frac{\omega_{n}^{2}+2 i \omega_{n} \gamma_{n}}{c^{2}}
$$

where $\omega_{n}$ and $\gamma_{n}$ are real. It follows that

$$
c^{2} k_{n}^{2}=\sum_{j=x, y, z} \omega_{n_{j}}^{2}+2 i \omega_{n_{j}} \gamma_{n_{j}}=\omega_{n}^{2}\left(1+\frac{2 i}{\omega_{n}^{2}} \sum_{j=x, y, z} \omega_{n_{j}} \gamma_{n_{j}}\right)
$$

with $\omega_{n}^{2}=\sum_{j=x, y, z} \omega_{n_{j}}^{2}$. Since $2 i / \omega_{n}^{2} \sum_{j=x, y, z} \omega_{n_{j}} \gamma_{n_{j}} \ll 1$, an approximate expression for $c k_{n}$ can be found by applying the series expansion of $(1+x)^{1 / 2}$ truncated after the first term, which gives

$$
\mathrm{c} k_{n} \approx \omega_{n}\left(1+\frac{i}{\omega_{n}^{2}} \sum_{j=x, y, z} \omega_{n_{j}} \gamma_{n_{j}}\right) .
$$

Besides, Eq. (26) yields

$$
k_{n_{j}}^{2}=\left(\frac{n_{j} \pi}{L_{j}}\right)^{2}+\frac{2 i n_{j} \pi k}{k_{n_{j}} L_{j}^{2}}\left(\frac{1}{\xi_{j, 0}}+\frac{1}{\xi_{j, L_{j}}}\right)-\frac{k^{2}}{k_{n_{j}}^{2} L_{j}^{2}}\left(\frac{1}{\xi_{j, 0}}+\frac{1}{\xi_{j, L_{j}}}\right)^{2},
$$

for $j=x, y, z$, respectively. In the special case of nearly rigid boundaries (i.e. $\left|\xi_{j, 0}\right| \gg 1$ and $\left.\left|\xi_{j, L_{j}}\right| \gg 1\right)$, one may write

$$
k_{n_{j}}^{2} \approx\left(\frac{n_{j} \pi}{L_{j}}\right)^{2}+\frac{2 i k}{L_{j}}\left(\frac{1}{\xi_{j, 0}}+\frac{1}{\xi_{j, L_{j}}}\right) .
$$

Recalling that $c^{2} k_{n_{j}}^{2}=\omega_{n_{j}}^{2}+2 i \omega_{n_{j}} \gamma_{n_{j}}$, and equating the imaginary part with that of Eq. (A12), we get:

$$
\omega_{n_{j}} \gamma_{n_{j}}=\frac{c^{2} k}{L_{j}}\left(\frac{1}{\xi_{j, 0}}+\frac{1}{\xi_{j, L_{j}}}\right)
$$

for $j=x, y, z$, respectively. It follows that Eq. (B10) yields 


$$
c k_{n} \approx \omega_{n}\left(1+\frac{i c}{\omega} \sum_{j=x, y, z} \frac{1}{L_{j}}\left(\frac{1}{\xi_{j, 0}}+\frac{1}{\xi_{j, L_{j}}}\right)\right)
$$

where we have assumed that $\omega=\omega_{n}$, under the assumption that only modes whose resonant frequencies are close to the actual frequency will contribute significantly to the modal sum (provided that we are not calculating the sound pressure very near the sound source). Finally, by equating the imaginary parts in Eq. (A14) and $k_{n}=\left(\omega_{n}+i \gamma_{n}\right) / c$, we get

$$
\gamma_{n} \approx c \sum_{j=x, y, z} \frac{1}{L_{j}}\left(\frac{1}{\xi_{j, 0}}+\frac{1}{\xi_{j, L_{j}}}\right) .
$$

This equation can also be derived from equations already published in the literature. $\mathbf{8 , 9 , 1 2}$

\section{REFERENCES}

1. R. V. Waterhouse, "Statistical properties of reverberant sound fields," J. Acoust. Soc. Am. 43, 1436-1444 (1968).

2. M. Schroeder, "Die statistischen Parameter der Frequenzkurven von großen Raümen," Acustica 4, 594-600 (1954). [An English translation, "Statistical parameters of the frequency response curves of large rooms," has been published in Journal of the Audio Engineering Society 35, 299305 (1987)].

3. ISO 354:2003, "Measurement of sound absorption in a reverberation room" (International Organization for Standardization, Geneva, Switzerland, 2003), p.24.

4. R. H. Lyon, "Statistical analysis of power injection and response averaging in structures and rooms,” J. Acoust. Soc. Am. 45, 545-565 (1969).

5. J. L. Davy, "The relative variance of the transmission function of a reverberation room", J. Sound Vib. 77, 455-479 (1981).

6. R. L. Weaver, "On the ensemble variance of reverberation room transmission functions, the effect of spectral rigidity", J. Sound Vib. 130, 487-491 (1989). 
7. J. L. Davy, "The variance of the discrete frequency transmission function of a reverberation room," J. Acoust. Soc. Am. 126, 1199-1206 (2009).

8. P. M. Morse and R. H. Bolt, "Sound waves in rooms", Rev. Mod. Phys. 16, 61-150 (1944).

9. H. Kuttruff, "Room Acoustics” (Fifth Edition, Spon Press, 2009), pp. 374.

10. J. Kergomard, V. Debut and D. Matignon, "Resonance modes in a one-dimensional medium with two purely resistive boundaries: Calculation methods, orthogonality and completeness", J. Acoust. Soc. Am. 119, 1356-1367 (2006).

11. J. Leader and J. Pan, "On the completeness and orthogonality of the acoustic modes of an open cavity," in Proceedings of Acoustics 2015, Australian Acoustical Society (Hunter Valley, Australia), p.10.

12. P. M. Morse and K. U. Ingard, "Theoretical Acoustics” (McGraw-Hill, 1968), pp. 927.

13. R. L. Herman, "Strum-Liouville eigenvalues problems," in A second course in ordinary differential equations: dynamical systems and boundary value problems (2008) p. 185-203.

14. F. Jacobsen, "Sound power determination in reverberation rooms", Report No. 26, (The Acoustics Laboratory, Technical University of Denmark, Kongens Lyngby, Denmark, 1979), pp. 111.

15. M. Abramowitz and I.A. Stegun, "Handbook of mathematical functions" (U.S. Department of Commerce, National Bureau of Standards, Applied Mathematics Series 55, Third printing, March 1965), pp. 1046.

16. J. L. Davy, "The spatial variability of random noise in a reverberant space", Proceedings of the 24th International Congress on Sound and Vibration, London (2017), pp. 8.

17. A. R. Conn, N. I. M. Gould and P. L. Toint, Trust Region Methods (Society of Industrial and Applied Mathematics, 2000), pp. 959.

18. https://www.mathworks.com/help/optim/ug/equation-solving-algorithms.html\#brnpdsm. 
19. F. V. Hunt, L. L. Beranek and D. Y. Maa, "Analysis of sound decay in rectangular rooms," J. Acoust. Soc. Am. 11(1), 80-94 (1939).

20. E. Nilsson, "Decay processes in rooms with non-diffuse sound fields. Part I: Ceiling treatment with absorbing material," Building Acoustic 11(1), 39-60 (2004).

21. https://www.comsol.com/release/5.3a.

22. F. Ihlenburg, Applied Mathematical Sciences, Vol. 132 (Springer-Verlag, New York, 1998).

23. M. Nolan and E. Fernandez-Grande, "Volumetric reconstruction of acoustic energy flows in a reverberation room,” J. Acoust. Soc. Am. 145(3), EL203-EL208 (2019).

24. F. Jacobsen, “Active and reactive, coherent and incoherent sound fields," J. Sound Vib. 130(3), 493-507 (1989).

25. F. Jacobsen, "A note on instantaneous and time-averaged active and reactive sound intensity," J. Sound Vib. 147(3), 489-496 (1991).

26. J. F. Fahy, "4.8.5 Sound fields in enclosures: the diffuse field," in Sound intensity (CRC Press, 1995), p. 72-77.

27. F. Jacobsen and A. Rodriguez Molares, "Ensemble statistics of active and reactive sound intensity in reverberation rooms," J. Acoust. Soc. Am. 129(1), 211-218 (2011).

\section{LIST OF FIGURES}

Fig.1: [Color online] Sound pressure level [dB SPL] at $70 \mathrm{~Hz}([1,0,1]$ mode) displayed as orthogonal slice planes in the $x$-, $y$ - and $z$ - direction, respectively, when (left) all boundaries are nearly rigid; (right) the floor is covered with highly absorptive material $\left(\alpha_{z=0}=0.9\right)$. Maximum order of modes included in the expansion: 15. Results are compared with finite element model (FEM) calculations.

Fig.2: Spatial standard deviation $s$ of the sound pressure level (in $[\mathrm{dB}]$ ) for different floor absorptions $\left(\alpha_{z=0}=0.9, \alpha_{z=0}=0.3\right.$ and a nearly rigid floor, respectively from left to right $)$. Numerical results 
(solid lines) are compared with theoretical predictions (dashed lines). Maximum order of modes included in the expansion: 15 .

Fig.3: [Color online] Active intensity field (magnitude in $\mathrm{W} / \mathrm{m}^{2}$ ) for the third-octave band centered at $125 \mathrm{~Hz}$ in the undamped (left) and damped (right) room $\left(\alpha_{z=0}=0.9\right)$. Maximum order of modes included in the expansion: 15 .

Fig.4: [Color online] Active (top) and reactive (bottom) intensity fields (magnitude in $\mathrm{W} / \mathrm{m}^{2}$ ) at $58 \mathrm{~Hz}$ $[(0,0,1)$ mode $]$ in the undamped (left) and damped (right) room. Maximum order of modes included in the expansion: 15 .

Fig.5: [Color online] Spatial average of the active and reactive intensity magnitudes (in $\mathrm{W} / \mathrm{m}^{2}$ ) in the (a) undamped and (b) damped room as a function of frequency (regions of low and high modal overlap are plotted separately). Maximum order of modes included in the expansion: 15 . 
Rigid floor
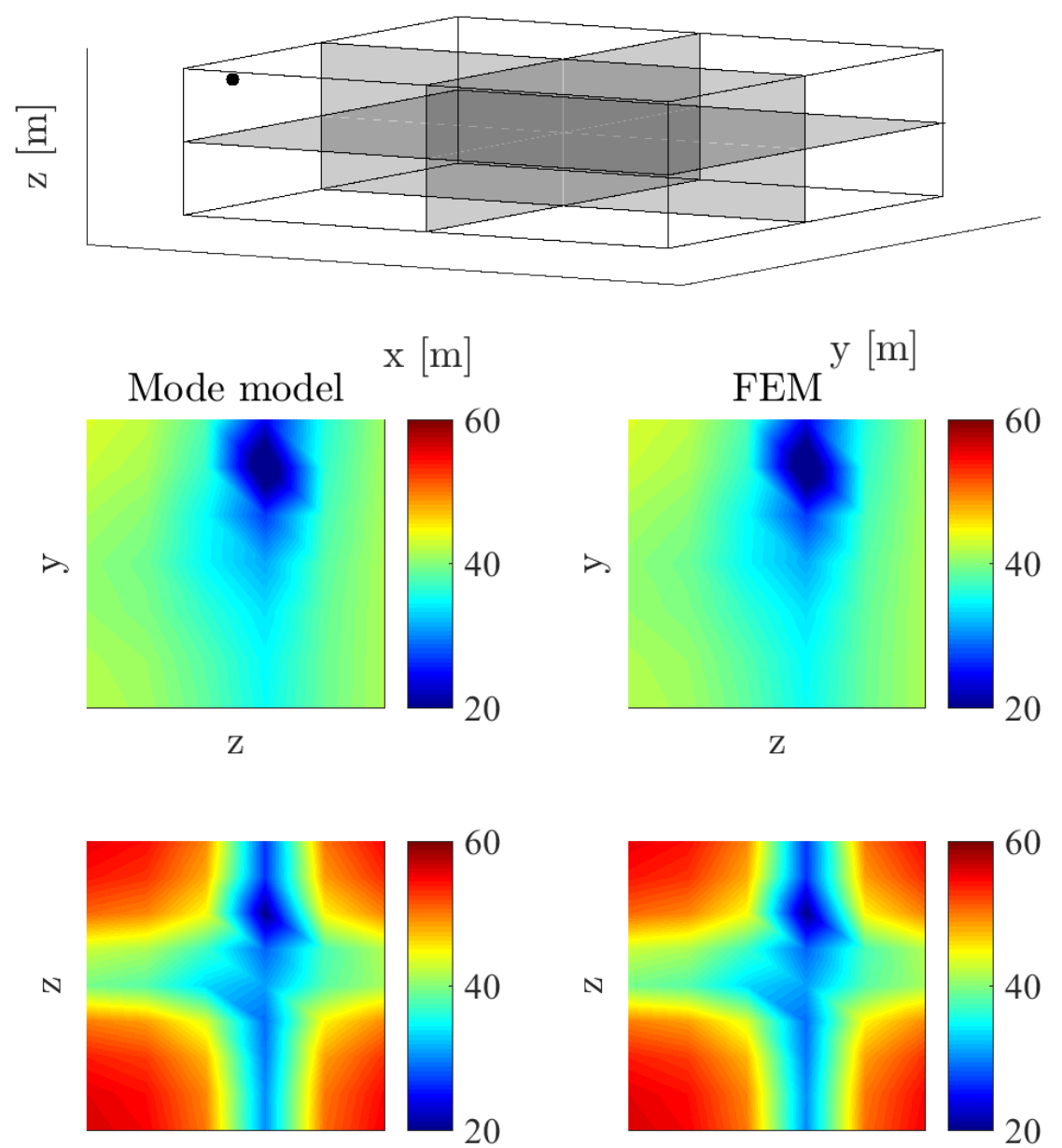

$\mathrm{X}$

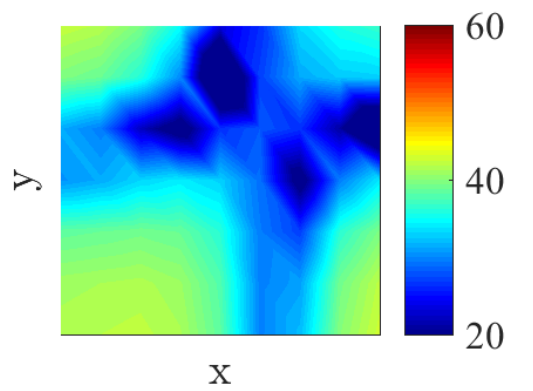

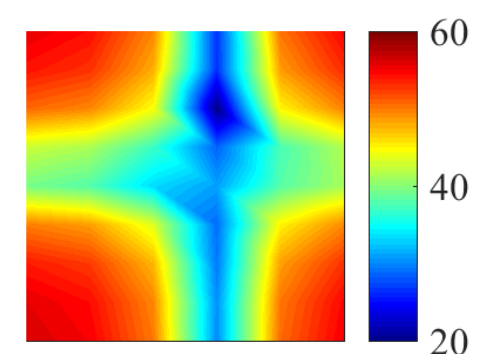

X

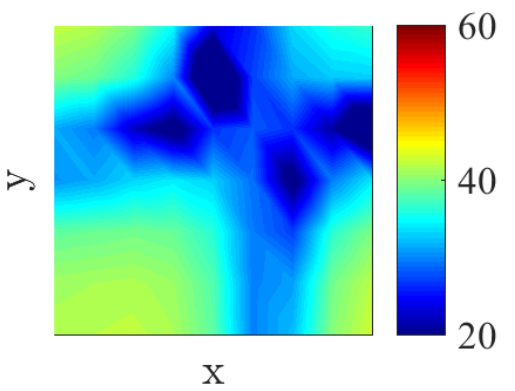

Absorptive floor
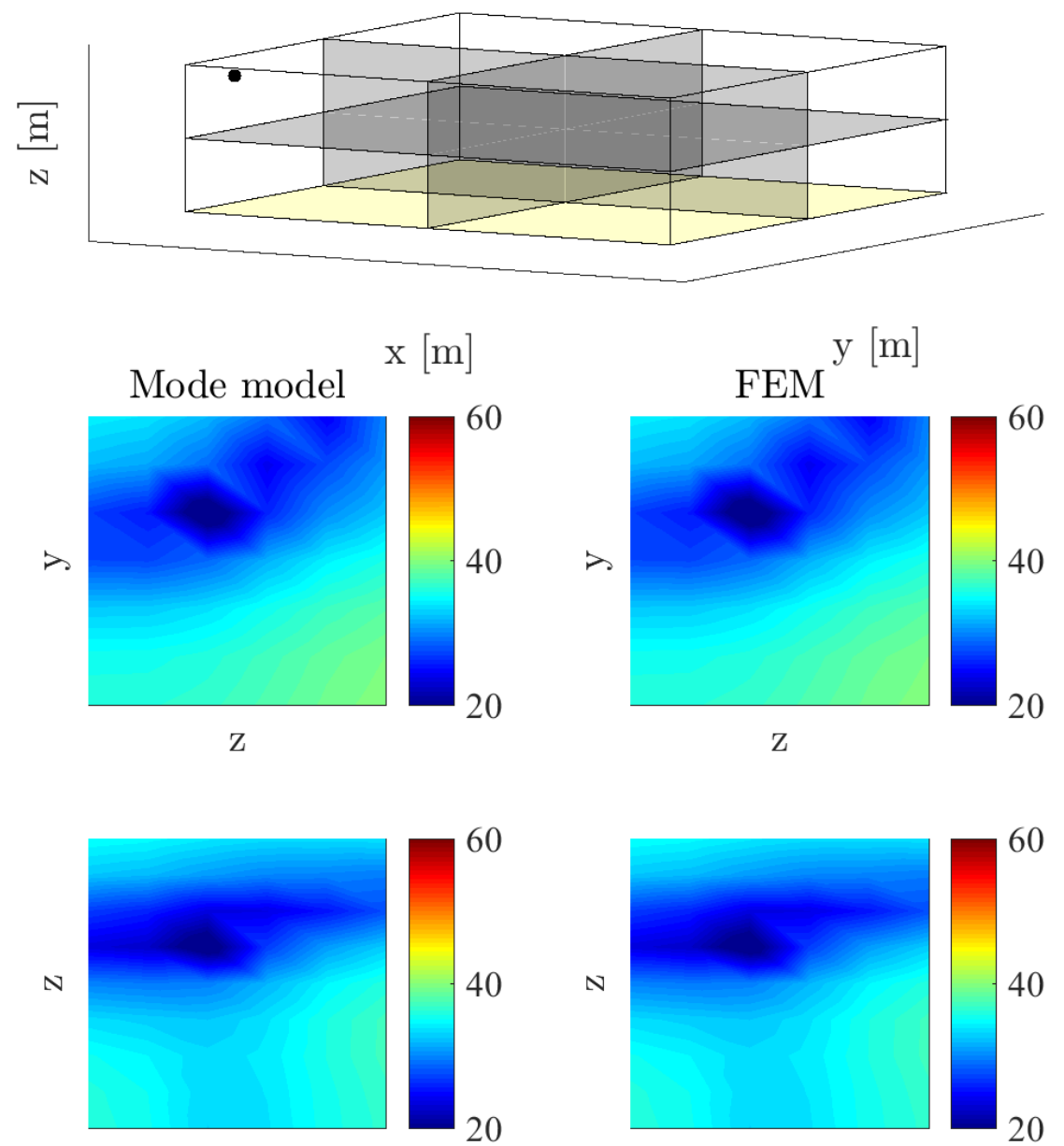

$\mathrm{X}$

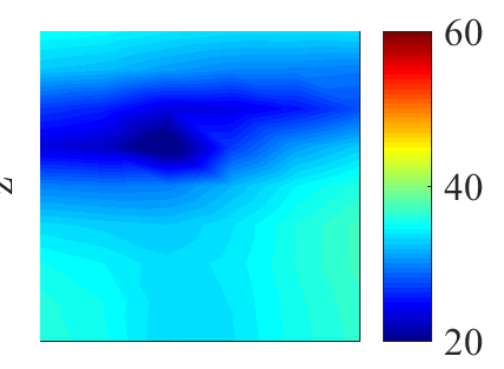

$\mathrm{x}$
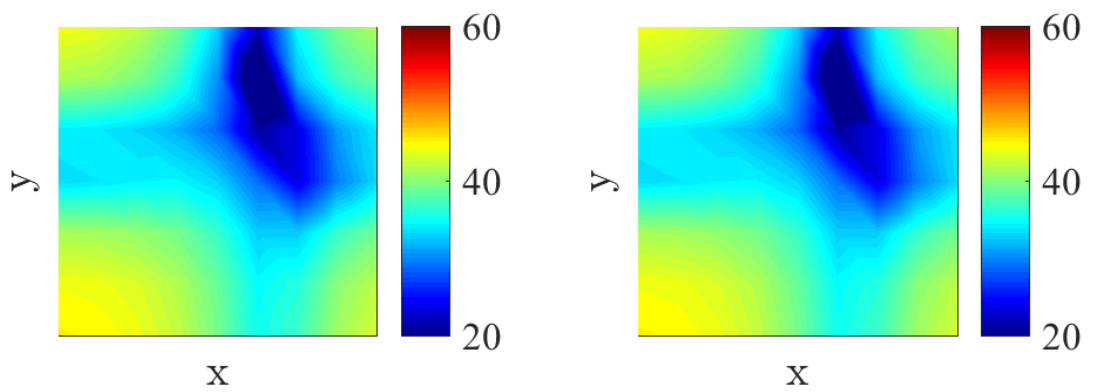

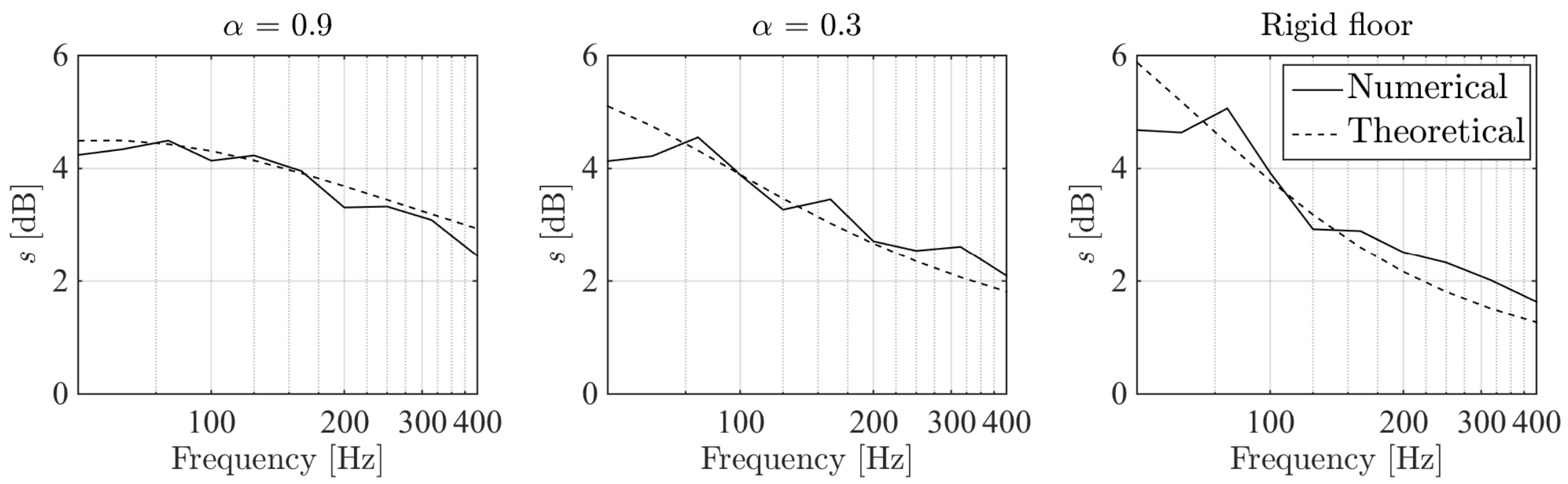

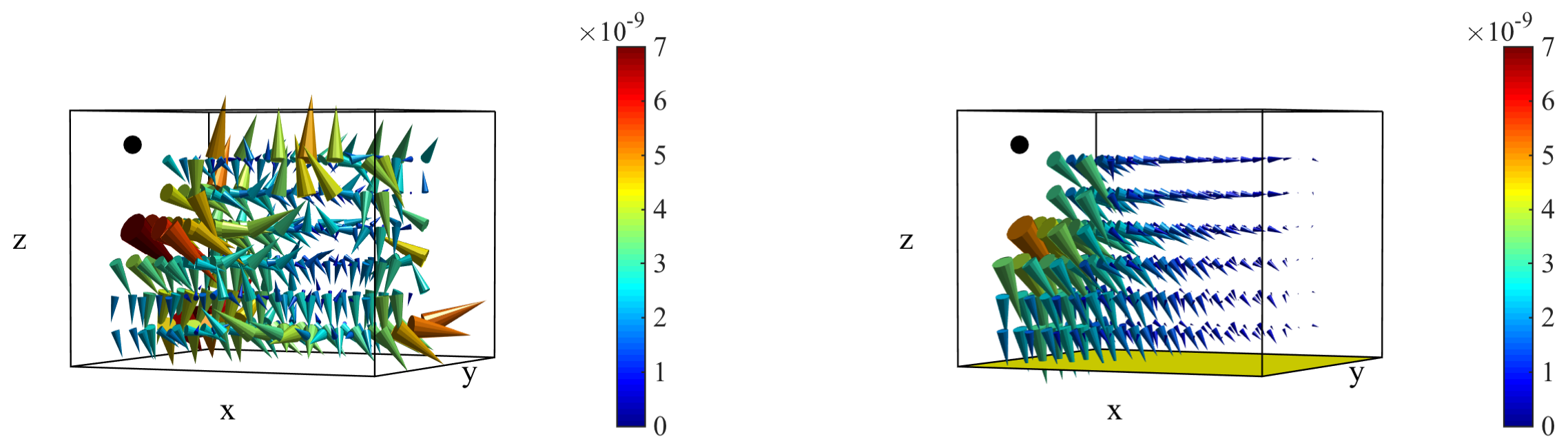

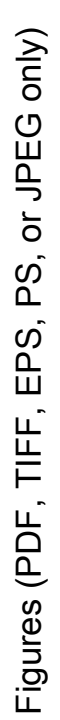



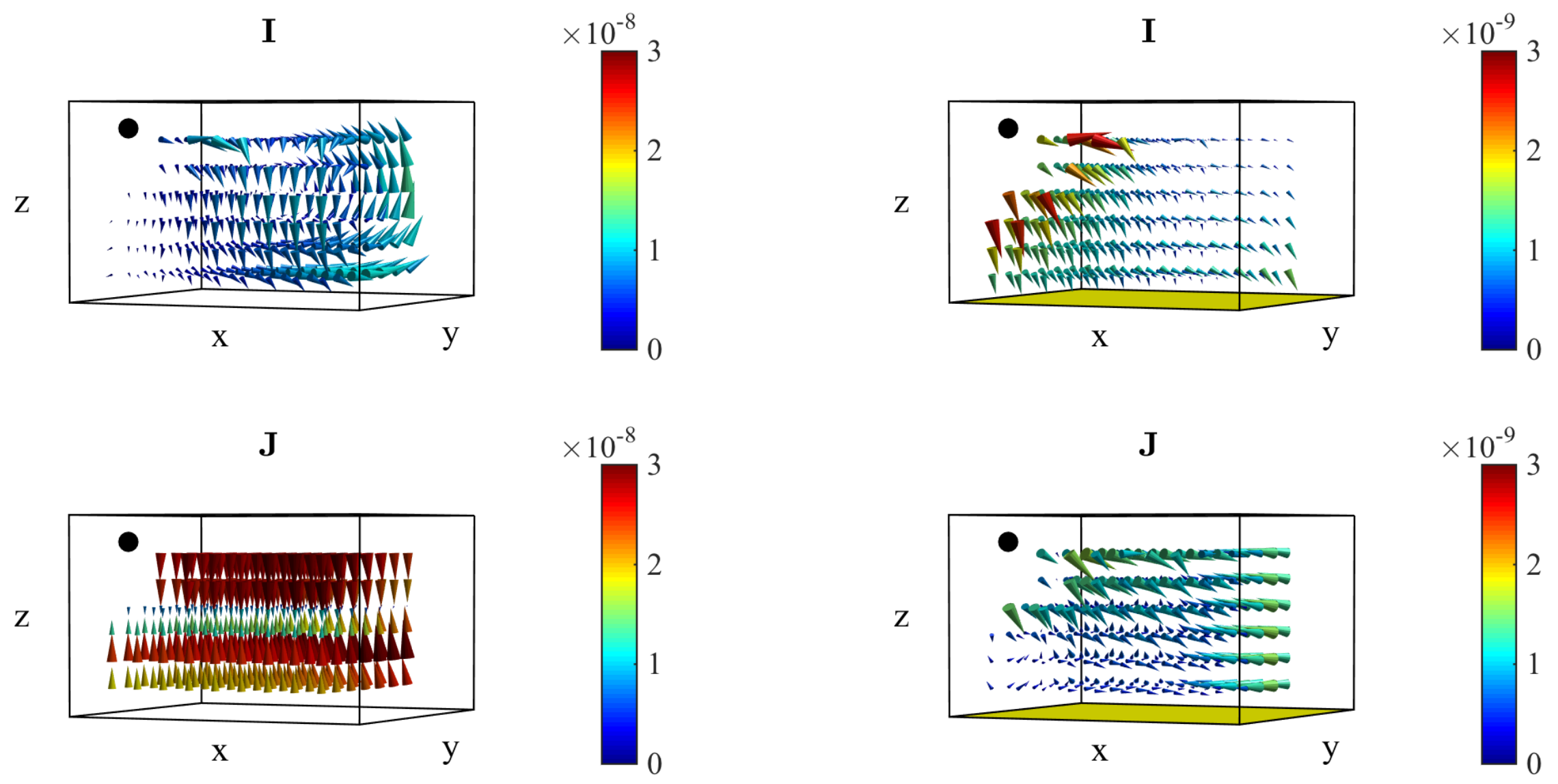
